\title{
A THEOREM ABOUT TOPOLOGICAL $n$-CELLS
}

M. K. FORT, JR.

1. Introduction. Let $R^{n}$ be Euclidean $n$-space. We define $I^{n}$ to be the set of all points $\left(x_{1}, \cdots, x_{n}\right)$ in $R^{n}$ such that $0 \leqq x_{j} \leqq 1$ for $j=1, \cdots, n$. Any set which is homeomorphic to $I^{n}$ will be called a topological $n$-cell. The result obtained in this paper is the following:

Theorem. If $G$ is a nonempty, open, connected subset of $R^{n}$, then there exists a nondecreasing sequence $E_{1} \subset E_{2} \subset E_{3} \subset \cdots$ of topological $n$-cells such that $\bigcup_{m=1}^{\infty} E_{m}=G$.

We point out that our topological $n$-cells are closed sets. It is obvious that in general $G$ could not be represented as the union of a nondecreasing sequence of "open topological $n$-cells." We also point out that we do not place any type of simple connectedness restriction on $G$.

The sets $E_{m}$ which we construct in the proof of our theorem are not only topological $n$-cells, but each is the union of a finite number of $n$-dimensional cubes. The same thing is true for the cells $E(i, j)$.

2. Proof of the theorem. If $k$ is a non-negative integer and $m_{1}, \cdots, m_{n}$ are integers, we define $\sigma\left(k ; m_{1}, \cdots, m_{n}\right)$ to be the set of all points $\left(x_{1}, \cdots, x_{n}\right)$ in $R^{n}$ for which $m_{j} 2^{-k} \leqq x_{j} \leqq\left(m_{j}+1\right) 2^{-k}$ for $j=1, \cdots, n$. We define $\Sigma$ to be the set of all $n$-cells $\sigma\left(k ; m_{1}, \cdots, m_{n}\right)$ which are contained in $G . \Sigma$ is countable and we arrange its members in a sequence $S_{1}, S_{2}, \cdots$.

We are going to define topological $n$-cells $E(i, j)$ for all positive integers $i, j$ in such a way that:

(1) $E(i, j) \subset E(i, j+1)$ and $E(i, j) \subset E(i+1, j)$ for all $i, j$;

(2) $P_{i}=\cup_{j=1}^{\infty} E(i, j)$ can be expressed as the union of a finite number of members of $\Sigma$;

(3) $S_{i} \subset P_{i}$ for each $i$.

Once we have defined sets $E(i, j)$ having the above properties, it is easy to see that $E(1,1) \subset E(2,2) \subset E(3,3) \subset \cdots$ and that $\bigcup_{m=1}^{\infty} E(m, m)=G$. Thus we can define $E_{m}=E(m, m)$ and obtain topological $n$-cells which have the desired properties.

We think of the sets $E(i, j)$ as being the elements of an infinite matrix, with $i$ being the "row variable" and $j$ being the "column variable." The sets $E(i, j)$ are to be defined a row at a time.

We need the following lemma.

Presented to the Society, November 27, 1953; received by the editors August 10, 1953. 
Lemma 1. Suppose that a row $E(m, 1), E(m, 2), \cdots$ has been defined in such a way that (1) and (2) are satisfied, and suppose that $J$ is an $(n-1)$-cell on the boundary of $P_{m}$. Then there exists an integer $k$ and an $(n-1)$-cell $J^{*}$ such that $J^{*} \subset J$ and $J^{*} \subset E(m, j)$ for all $j \geqq k$.

Proof. Let $C(j)=J \cap E(m, j)$ for each positive integer $j$. Then each $C(j)$ is a closed subset of $J$. Since $J=\cup_{j=1}^{\infty} C(j)$ and $J$ is of the second category with respect to itself, there exists an integer $k$ such that $C(k)$ contains an open set relative to $J$. We may choose $J^{*}$ to be any $(n-1)$-cell which is contained in this open set.

We begin the definitions of the sets $E(i, j)$ by defining $E(1, j)=S_{1}$ for each positive integer $j$.

Now assume that $m>1$ and that for all $i<m$ we have defined the topological $n$-cells $E(i, j)$ for all $j$, and that these sets have been defined in such a way that (1), (2), and (3) are satisfied. We wish to define next sets $E(m, 1), E(m, 2), \cdots$. There are five cases which we consider.

Case 1. $S_{m} \cap P_{m-1}=\varnothing$.

Let $C$ be the component of $G-P_{m-1}$ that contains $S_{m}$, and let $J$ be an $(n-1)$-cell which is contained in $\bar{C} \cap P_{m-1}$. We use the lemma to obtain an integer $k$ and an $(n-1)$-cell $J^{*} \subset J$ such that $J^{*} \subset E(m-1, j)$ for all $j \geqq k$. It is easy to see that we can unite a finite number of members of $\Sigma$ to form a topological $n$-cell $F$ such that: $F \subset \bar{C}, F \cap P_{m-1}$ is an $(n-1)$-cell which is contained in $J^{*}$, and $F \cap S_{m}$ is an $(n-1)$-cell. It follows that $E(m-1, j) \cup F \cup S_{m}$ is a topological $n$-cell for $j \geqq k$. We define $E(m, j)=E(m-1, j)$ for $j<k$ and $E(m, j)=E(m-1, j)$ $\cup F \cup S_{m}$ for $j \geqq k$.

Case 2. $P_{m-1}$ does not contain interior points of $S_{m}$, and $P_{m-1} \cap S_{m}$ contains some $(n-1)$-cell $J$.

We again use the lemma to obtain an integer $k$ and an $(n-1)$-cell $J^{*}$ such that $J^{*} \subset J \cap E(m-1, j)$ for all $j \geqq k$.

If $\epsilon$ is a positive number of the form $2^{-q}, q$ a positive integer, we define $S_{m}(\epsilon)$ to be the closure of the set obtained by subtracting from $S_{m}$ all members of $\Sigma$ which have edges of length $\epsilon$ and which contain points of $S_{m} \cap P_{m-1}$. It is obvious that for sufficiently small $\epsilon, S_{m}(\epsilon)$ is a topological $n$-cell. We assume that $\delta=2^{-q}$ is so small that $S_{m}(\epsilon)$ is a topological $n$-cell for all $\epsilon<\delta$.

It is possible to unite a finite number of members of $\Sigma$ to form a topological $n$-cell $F$ for which: $F \subset S_{m}, F \cup S_{m}(\epsilon)$ is a topological $n$-cell for $\epsilon<\delta, F \cap P_{m-1}$ is a topological $(n-1)$-cell which is contained in $J^{*}$. It follows that $E(m-1, j) \cup F \cup S_{m}\left(2^{-j} \delta\right)$ is a topological $n$-cell for $j \geqq k$. We define $E(m, j)=E(m-1, j)$ for $j<k$ and we define $E(m, j)=E(m-1, j) \cup F \cup S_{m}\left(2^{-j} \delta\right)$ for $j \geqq k$. 
Case 3. $P_{m-1}$ does not contain interior points of $S_{m}$, and $S_{m} \cap P_{m-1}$ contains only cells of dimension less than $n-1$.

We choose an $(n-1)$-cell $J$ contained in the intersection of $P_{m-1}$ with the boundary of the component $C$ of $G-P_{m-1}$ which contains the interior of $S_{m}$. We also require that $J$ be disjoint from $S_{m} \cap P_{m-1}$. An integer $k$ and an $(n-1)$-cell $J^{*}$ are chosen as in case 2 , and sets $S_{m}(\epsilon)$ are defined as in case 2 . We also choose $\delta$ as in case 2 . It is possible to unite a finite number of members of $\Sigma$ so as to form a topological $n$-cell $F$ such that $F \cup S_{m}(\epsilon)$ is a topological $n$-cell for all $\epsilon<\delta$ and such that $F \cap P_{m-1}$ is an $(n-1)$-cell which is contained in $J^{*}$. We define the sets $E(m, j)$ as in case 2 .

Case 4. $P_{m-1}$ contains interior points of $S_{m}$, but does not contain all of $S_{m}$.

It is easy to see that the closure of $S_{m}-P_{m-1}$ can be expressed as the union of a finite set $\sigma_{1}, \cdots, \sigma_{p}$ of elements of $\Sigma$. We start with the sequence $E(m-1,1), E(m-1,2), \cdots$ and apply the construction outlined in case $2 p$ times to construct successively $p$ sequences of topological $n$-cells. This may be done in such a way that the union of the elements of the $j$ th sequence constructed will contain $j$ members of the set $\sigma_{1}, \cdots, \sigma_{p}$. We take the last of the $p$ sequences which we construct to be the sequence $E(m, 1), E(m, 2), \cdots$. It is easily seen that $S_{m} \subset \cup_{j=1}^{\infty} E(m, j)$.

Case 5. $S_{m} \subset P_{m-1}$.

We define $E(m, j)=E(m-1, j)$ for all $j$.

In the following corollary, $\Pi_{j}(G-H)$ is the $j$ th homotopy group of $G-H$.

Corollary 1. If $G$ is a nonemply, open, connected subset of $R^{n}$, then there exists a subset $H$ of $G$ such that the dimension of $H$ is less than $n, G-H$ is open and connected, and $\Pi_{j}(G-H)=0$ for all $j$.

Proof. Let $E_{1} \subset E_{2} \subset E_{3} \subset \cdots$ be topological $n$-cells such that $\bigcup_{m=1}^{\infty} E_{m}=G$. We let $B_{m}$ be the boundary of $E_{m}$ and let $U_{m}=E_{m}-B_{m}$. We define $H=\bigcup_{m=1}^{\infty} \bigcap_{j=m}^{\infty} B_{j}$.

Since each $B_{j}$ is closed and of dimension $n-1, \bigcap_{j=m}^{\infty} B_{j}$ is closed and of dimension less than $n$ for each $m$. It follows from the theorem on countable unions of closed sets that $H$ is also of dimension less than $n$.

It is easy to verify that $G-H=\bigcup_{m=1}^{\infty} U_{m}$. Since each $U_{m}$ is open and connected and $U_{1} \subset U_{2} \subset U_{3} \subset \cdots, G-H$ is also open and connected.

Let $S$ be a $j$-sphere, and let $f$ be a continuous function on $S$ into $G-H$. Then $f(S)$ is compact, and it follows that $f(S) \subset U_{m}$ for some $m$. Since $U_{m}$ is homeomorphic to an open $n$-cell in $R^{n}, f$ is homotopic 
to a constant in $U_{m}$, and hence also in $G-H$. Thus $\Pi_{j}(G-H)=0$.

For the special case of open connected subsets of $R^{n}, n \leqq 3$, we can strengthen Corollary 1 and show that the set $H$ can be chosen so that $G-H$ is an open topological 3-cell. Since the result is relatively trivial for $n=2$ and is completely trivial for $n=1$, we restrict our attention to the case $n=3$. We use a theorem of Moise to prove:

LEMmA 2. If $I^{\prime}$ and $I^{\prime \prime}$ are closed 3-cells, $J^{\prime}$ and $J^{\prime \prime}$ are closed polyhedral 3-cells, $I^{\prime}$ is a subset of the interior of $I^{\prime \prime}, J^{\prime}$ is a subset of the interior of $J^{\prime \prime}$, and $h$ is a piecewise linear homeomorphism of $I^{\prime}$ onto $J^{\prime}$; then, $h$ can be extended to a piecewise linear homeomorphism which maps $I^{\prime \prime}$ onto $J^{\prime \prime}$.

Proof. There exists a piecewise linear homeomorphism $k$ of $I^{\prime \prime}$ on to $J^{\prime \prime}$. We define $A=k^{-1} h\left(I^{\prime}\right)$. It follows from a theorem of Moise (see [1, Theorem 1]) that there exists a piecewise linear homeomorphism $\phi$ of $I^{\prime \prime}$ onto $I^{\prime \prime}$ which takes $A$ onto $I^{\prime}$. It is easy to see that $\phi k^{-1} h$ maps $I^{\prime}$ onto $I^{\prime}$, and can thus be extended in an obvious manner to a piecewise linear homeomorphism $\psi$ of $I^{\prime \prime}$ onto $I^{\prime \prime}$. We define $h^{*}=k \phi^{-1} \psi$. Then $h^{*}$ is a piecewise linear homeomorphism which is an extension of $h$ and which maps $I^{\prime \prime}$ onto $J^{\prime \prime}$.

CoRollary 2. If $G$ is an open, connected subset of $R^{3}$, then there exists a subset $H$ of $G$ of dimension less than 3 for which there exists a piecewise linear homeomorphism of $R^{3}$ onto $G-H$.

Proof. It follows from our theorem (and its proof) that there exist closed polyhedral 3-cells $E_{1} \subset E_{2} \subset \cdots$ such that $\bigcup_{m=1}^{\infty} E_{m}=G$. We define $U_{m}, B_{m}$, and $H$ as in Corollary 1 . It is easily seen that there exist closed polyhedral 3-cells $J_{m} \subset U_{m}$ such that $\cup_{m=1}^{\infty} J_{m}=\cup_{m=1}^{\infty} U_{m}$ $=G-H$ and such that $J_{m}$ is a subset of the interior of $J_{m+1}$ for each $m$.

We define $I_{m}$ to be the set of all points $\left(x_{1}, x_{2}, x_{3}\right)$ in $R^{3}$ for which $\max \left(\left|x_{1}\right|,\left|x_{2}\right|,\left|x_{3}\right|\right) \leqq m$. There exists a piecewise linear homeomorphism $h_{1}$ of $I_{1}$ onto $J_{1}$. If $h_{m}$ has been defined so as to be a piecewise linear homeomorphism of $I_{m}$ onto $J_{m}$, then we use Lemma 2 to extend $h_{m}$ to a piecewise linear homeomorphism $h_{m+1}$ on $I_{m+1}$ onto $J_{m+1}$. If $x \in R_{3}$, then there exists $m$ such that $x \in I_{m}$ and we define $h(x)=h_{m}(x)$. The function $h$ is a piecewise linear homeomorphism of $R^{3}$ onto $G-H$.

\section{BIBLIOGRAPHY}

1. Edwin E. Moise, Affine structures in 3-manifolds II. Positional properties of 2-spheres, Ann. of Math. vol. 55 (1952) pp. 172-176.

The University of Georgia 